
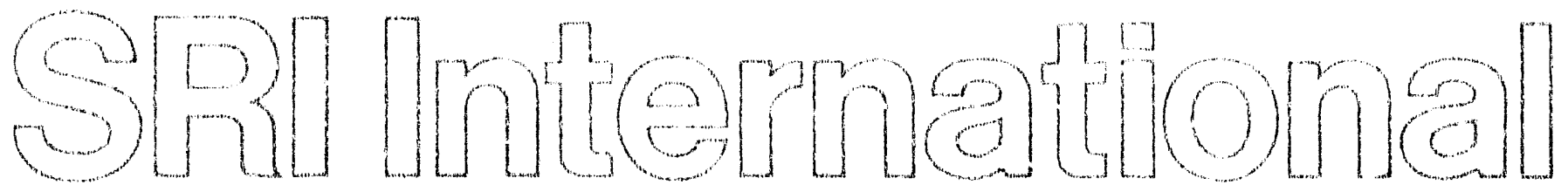

$\mathrm{DOE} / \mathrm{PC} / 90036--\mathrm{Tl}$

DE9 I 005598

\title{
SYNTHESIS OF MODEL COMPOUNDS FOR COAL LIQUEFACTION RESEARCH
}

By: Albert S. Hirschon, Marianna Asaro, and Jeffrey Bottaro

SRI Project 1285

Period Covering June 21, 1990 through September 20, 1990

Prepared for:

United States Department of Energy

Pittsburgh Energy Technology Center

P.O. Box 10940

Pittsburgh, PA 15236

Contract No. DE-AC22-90PC90036

\section{DISCLAIMER}

This report was prepared as an account of work sponsored by an agency of the Unitid States Government. Neither the United States Government nor any agency thereof, nor any of their employees, makes any warranty, express or implied, or assumes any legal liability or responsibility for the accuracy, completeness, or usefulness of any information, apparatus, ; roduct, or process disclosed, or represents that its use would not infringe privately nuned rights. Reference herein to any specific commercial product, process, or service by trade name, trademark, manufacturer, or otherwise does not necessarily constitute or imply its endorsernent, recommendation, or favoring by the United States Government or any agency thereof. The views and opinions of authors expressed herein do not necessarily state or reflect those of the United States Government or any agency thereof. 


\section{SUMMARY}

The objectives of this project are to develop feasible synthetic routes to produce (1) 4(4'-hydroxy-5',6',7',8'-tetrahydro-1'-naphthylmethyl)-6-methyldibenzothiophene, and (2) a 1-hydroxynaphthalene-dibenzothiophene polymer. These compounds are thought to be representative of sulfur containing molecules in coal. The program is divided into three tasks, the first of which is a project work plan that we have already submitted. Our experimental work during this quarter concentrated on Task 2 and is described below.

\section{Task 2. Synthesis of $4\left(4^{\prime}\right.$-hydroxy-5',6',7',8'-tetrahydro-1'-} naphthylmethyl)-6-methyldibenzothiophene

As discussed if our project work plan in Task 1, there are several possible synthetic routes to the target molecule, $4\left(4^{\prime}\right.$-hydroxy-5',6, $7^{\prime}, 8^{\prime}$-tetrahydro-1'-naphthylmethyl)-6methyidibenzothiophene. This quarter we concentrated on synthesizing the intermediates for our first method, in which we couple a metalated 4-methyldibenzothiophene with 4formyl-5,6,7,8-tetrahydro-1-naphthol. We found that we could easily metalate dibenzothiophene, and then add a methyl group to the 4-position to give 4methyldibenzothiophene in greater than $80 \%$ yield by using $t$-butyllithium in tetrahydropyran followed by dimethylsulfate. However, adding the second metal to the desired 4' position using the same method was more difficult, and instead the reaction occurred on the methyl group. Therefore, during the next quarter we will investigate an alternative method, in which a hydroxy group is added in order to help dinect the second metalation step to the 4 ' position on 4-methyldibenzothiophene. 


\section{INTRODUCTION}

To effectively liquefy coal and convert it into usable coal liquids, we need to study the fundamental reaction chemistry for the individual molecular linkages in the coal matrix. For instance, we need to find which linkages are important during coal liquefaction and which substituents on these groups either aid or retard bond breaking. Furthermore, in competitive reactions, we need to know how these functional groups affect each other in hydrogenolysis and hydrogenation chemistry. One method to understand this chemistry is to study molecules designed to represent coal molecules. Thus, the DOE wants synthetic methods for and samples of such coal models with various functional groups thought to be present in coal.

An important functional group in coal is that of sulfur. Given et al. have observed correlations between total sulfur and coal conversion. ${ }^{1,2}$ Hydrogen sulfide, and organic sulfur groups that easily cleave to form hydrogen sulfide, are well known to enhance coal liquefaction. ${ }^{3-9}$ Thus understanding the role of sulfur, whether organic or inorganic, and its fate is important to understanding coal liquefaction and pyrolysis mechanisms. The organic sulfur in coal is generally one-half to one-third of the total sulfur content, and the organic sulfur in bituminous coals is estimated to be composed mainly of thiophenes (40$70 \%$ ), with the remainder as sulfides. ${ }^{10}$ Sulfides are readily decomposed to liberate $\mathrm{H}_{2} \mathrm{~S}$, which aids in coal liquefaction. However, thiophenes are much more stable and readily formed. 10 For instance, iron pyrite reacts with certain hydrocarbons such as ethylene to form thiophenes. Thiophenes are also formed from reactions of organic molecules and $\mathrm{H}_{2} \mathrm{~S}$ or sulfur. The thiophene ring is reported to be stable up to $800^{\circ} \mathrm{C}$, but the alkyl groups may cleave at lower temperatures, such as $500^{\circ} \mathrm{C}$. Decomposition of dibenzothiophene is catalyzed by alumina or aluminosilicates. Once thiophenic structures are formed, it is very difficult to decompose or to hydrogenate them, and therefore a knowledge of the reactivities of thiophenes thought to be present in coal or coal liquids is essential to be able to remove the sulfur to produce clean fuels.

The purpose of this research is to develop syntheses for specific coal models and deliver samples of these compounds to the DOE to be presented to various researchers throughout the United States. These particular compounds include sulfur heterocyclic monomers and polymers containing linkages of oxygen, methylene, and ethylene as well as 
phenolics. These models also contain hydrogen donor functionalities. Thus, these compounds will allow researchers to compare hydrogenolysis reactions of methylene, ethylene linkages, and hydrodesulfurization, hydrodeoxygenation, hydrogen transfer, and hydrogenation reactions.

The goal of this work is to develop syntheses for and supply of one-pound samples of the above sulfur containing compounds thought to be present in coal for coal liquefaction studies. These model compounds are to be packaged in small quantities for long-term storage and delivered to the DOE PETC, which will make them available to researchers throughout the United States for studies of the fundamental reaction chemistry of the individual molecular links in the coal matrix. From these models, researchers can systematically determine the role of each type of bond and functional group for coal liquefaction and pyrolysis. 


\section{TASK 2. SYNTHESIS OF 4(4'-HYDROXY-5',6',7',8'- TETRAHYDRO-1'-NAPHTHYLMETHYL)-6" METHYLDIBENZOTHIOPHENE}

The objective of this task is to develop a facile synthesis for the coal model 4(4'hydroxy-5',6',7',8'-tetrahydro-1'-naphthylmethyl)-6-methyldibenzothiophene. As discussed in the project work plan, there are several potential routes to the synthesis of the target molecule. The choice of final synthetic route for the large scale reaction will depend on the relative number of isomers and any isolation procedures needed. This quarter we investigated metalation reactions on dibenzothiophene and preparation of intermediates for the coupling reaction, as described below.

\section{APPROACH}

Our synthesis approach to the unsymmetrical, disubstituted dibenzothiophene derivative 1 involves sequential metalation steps. Dibenzothiophene is first mono-metalated with t-butyl lithium, the resultant anion is functionalized, and then the 4-substituted dibenzothiophene is mono-metalated in the 4' position as illustrated in reaction 1. Although the powerful base $t$-butyl lithium performs the deprotonation well, we also investigated the use of the less expensive and milder $n$-butyl lithium reagent. This intermediate (1) can then be coupled as shown in reaction 2 to give the carbon skeleton of the desired product $\underline{A}$, which can be prepared by simple hydrogenation of the intermediate coupled product.

\section{EXPERIMENTAL}

Metalation of dibenzothiophene. The following is a typical experimental procedure for the metalation and subsequent alkylation of tetrahydrothiophene. Dibenzothiophene dissolved in either 1,2-dimethoxyethane or tetrahydropyran was treated with 1 to 1.25 equivalents of $\mathrm{n}$-butyl lithium or $\mathrm{t}$-butyl lithium under an inert atmosphere at temperatures ranging from $5^{\circ} \mathrm{C}$ to $25^{\circ} \mathrm{C}$. The metalation was performed with and without added $N, N^{\prime}$, N", N"'-tetramethylethylene diamine. The mixture was stirred for 20 minutes to one hour and then methylated using either dimethylsulfate, methyl iodide, or trimethyloxonium tetrafluoroborate at ambient temperature. Crude yields of 4-methyidibenzothiophene 
ranged from $55 \%$ for the reaction with n-butyl lithium to $84 \%$ with $t$-butyl lithium as determined by ${ }^{1}$ H NMR spectroscopy. The yield of the dimethylated product, 4,4'dimethyldibenzothiophene, was $7 \%$ for the reaction with $t$-butyl lithium. In contrast, when 4-methyldibenzothiophene was metalated a second time, the predominate methylation was on the 4 , rather than the 4 ' position. This contingency will require development of a new metalation scheme.

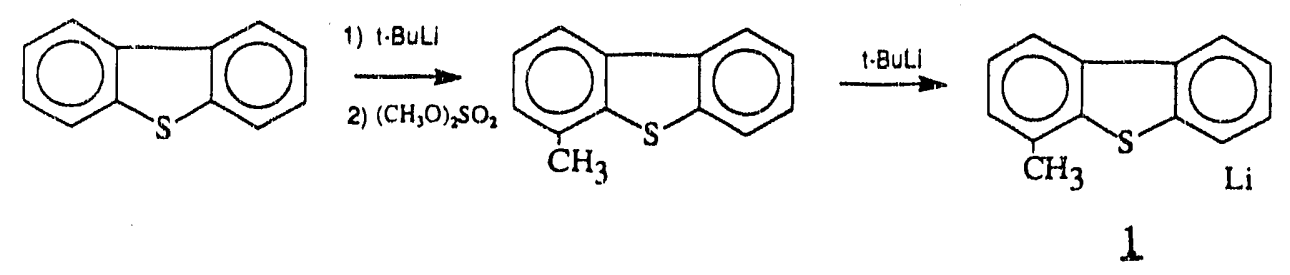

Eormylation of 5.6.7.8-tetrahydro-1-naphthol. The formylation of 5,6,7,8tetrahydro-1-naphthol was attempted using the Vilsmeier reagent made from $\mathrm{N}, \mathrm{N}$ dimethylformarnide (DMF) and either phosphorous oxychloride or phosgene. The Vilsmeier reagent was generated either at $0^{\circ} \mathrm{C}$ from $\mathrm{POCl}_{3}$ or at ambient temperature from $\mathrm{COCl}_{2}$. The phenol was added in either $\mathrm{DMF}$ or $\mathrm{CHCl}_{3}$ and the mixture refluxed for several hours. The reaction was quenched with water, made basic $(\mathrm{pH} \geq 12)$ with $\mathrm{NaOH}$, and refluxed for several hours. Acidification of the mixture with aqueous $\mathrm{HCl}$ followed by extraction with hexane yielded only the starting material, based on ${ }^{1} \mathrm{H}$ NMR spectroscopy. In one run, a reaction intermediate was isolated by quenching with water, rendering the mixture only slightly basic ( $\mathrm{pH}$ ca. 8) without refluxing, and finally extracting with $\mathrm{CH}_{2} \mathrm{Cl}_{2}$. This intermediate was subsequently converted to the starting material by stirring with aqeous $\mathrm{KOH}$ at room temperature. This unstable intermediate was identified by ${ }^{1} \mathrm{H}$ NMR and IR spectroscopy as the formate ester of the phenol. 
<smiles>Cc1cccc2c1sc1c([Al])cccc12</smiles><smiles>O=Cc1ccc(O)c2c1CCCC2</smiles><smiles>CCCC</smiles><smiles>Cc1cccc2c1sc1c(O)cccc12</smiles>

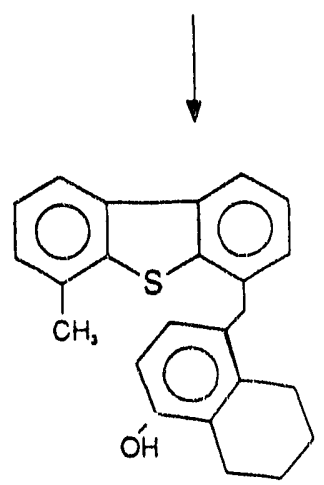

A

\section{RESULTS AND DISCUSSION}

We feel that the lack of formylation on the ring by the Vilsmeier reaction can easily be overcome. This reaction requires that an activating substituent such as amino or hydroxyl be present on the aromatic ring. In this example, however, the formyl group apparently is not transferred from the hydroxyl group to the ring. Protection of the phenol, for example as an ether, would avoid formation of the formate ester but also probably would deactivate the ring. If we need to pursue this synthesis route further; we are aware of a synthesis of 4-formyl-5,6,7,8-tetrahydro-1-naphthol by the Gattermann method using zinc cyanide, albeit in rather low yield. ${ }^{11}$ 
However, next quarter we plan an alternate approach to this synthesis in which we introduce a COII group onto the dibenzothiophene as shown in Figure 3. We believe that this sequence of metalations and functionalizations is the key to the preparation of the unsymmetrical thiophene target molecule. The hydroxy group is very important, as its deprotonation forces the subsequent deprotonation on the thiophene ring, rather than on the alkyl group. Our initial results appear promising, and we will pursue this approach during the next quarter.<smiles>c1ccc2c(c1)sc1ccccc12</smiles>

1) $\underset{\text { 2) } \mathrm{CO}_{2}}{\longrightarrow}$ 3) $\mathrm{LAIH}_{4}$

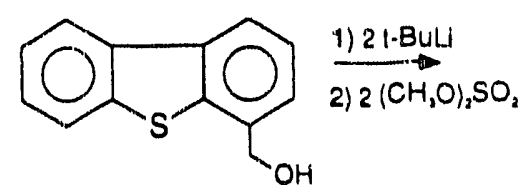<smiles>COCc1cccc2c1sc1c(C)cccc12</smiles>

(3) 


\section{REFERENCES}

1. M. B. Abdel-Basetg, R. F. Yarzab, and P. H. Given, Fuel (1978), 57, 89.

2. R. F. Yarzab, P. H. Given, W. Spackman, and A. Davis, Fuel (1980), 59, 81.

3. R. M. Baldwin and S. Vinciguerra, Fuel (1983), 62, 498.

4. V. I. Stenberg, V. R. Srinivas, P. Sweeny, R. J. Baltisberger, and N. F. Woolsey, Fuel (1983), 62, 913.

5. V. I. Stenberg. T. Ogawa, K. Raman, W. G. Wilson, and D. Miller, Fuel (1983), 62, 1487.

6. E. A. Sondreal, W. G. Wilson, and V. I. Stenberg, Fuel (1982), 61, 925.

7. H. R. Appel, and I. Wender, Am. Chem. Soc. Div. Fuel Chem. Prepr. (1968), 12, 220.

8. A. S. Hirschon and R. M. L aine, Fuel (1985), 64, 911.

9. M. J. Trewhella and A. Grint, Fuel (1987), 66, 1315.

10. A. Attar, Fuel (1978), 57, 201.

11. H. Wynberg and W. S. Johnson, J. Org. Chem. (1959) 24, 1424. 

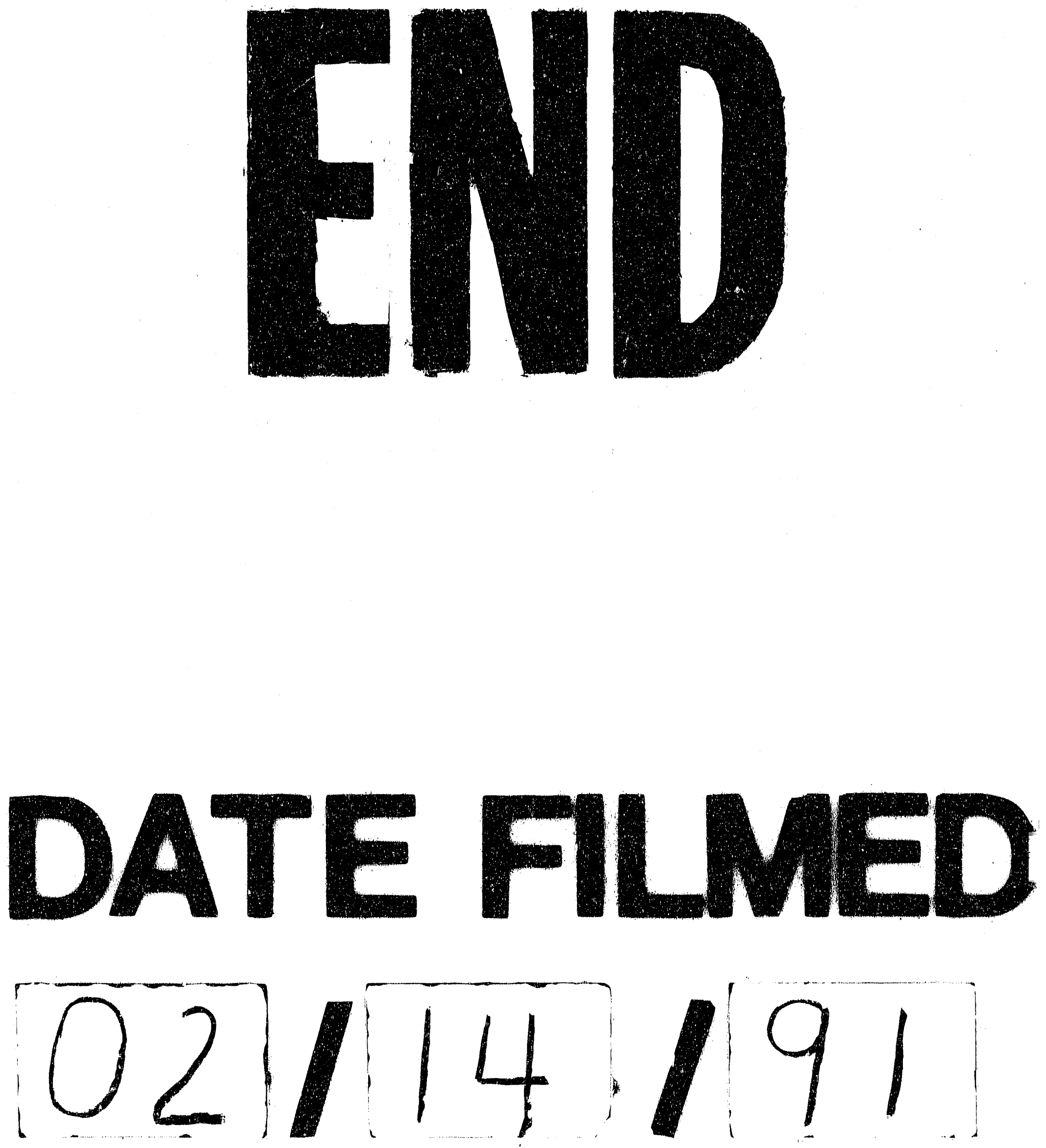
